\title{
Low Cost Virtual Face Performance Capture Using Stereo Web Cameras
}

\author{
Alexander Woodward ${ }^{1}$, Patrice Delmas ${ }^{1}$, Georgy Gimel'farb ${ }^{1}$, \\ and Jorge Marquez ${ }^{2}$ \\ ${ }^{1}$ Department of Computer Science, The University of Auckland, Auckland, \\ New Zealand \\ awoo016@ec.auckland.ac.nz, \{p.delmas,g.gimelfarb\}@auckland.ac.nz \\ ${ }^{2}$ Image Analysis Visualization Laboratory, CCADET UNAM, Mexico \\ marquez@aleph. cinstrum. unam.mx
}

\begin{abstract}
A complete system for creating the performance of a virtual character is described. Stereo web-cameras perform marker based motion capture to obtain rigid head motion and non-rigid facial expression motion. Acquired 3D points are then mapped onto a 3D face model with a virtual muscle animation to create face expressions. Muscle inverse kinematics updates muscle contraction parameters based on marker motion to create the character's performance. Advantages of the system are reduced character creation time by using virtual muscles and a dynamic skin model, a novel way of applying markers to a face animation system, and its low cost hardware requirements, capable of running on standard hardware and making it suitable for interactive media in end-user environments.
\end{abstract}

Keywords: Computer Vision, Web-camera, Markers, Motion capture, Facial animation, Virtual character.

\section{Introduction}

This paper presents a complete system for creating the performance of a virtual character. The system uses stereo web-cameras to perform marker based motion capture on a subject's face. 3D marker positions allow the derivation of rigid head motion and non-rigid facial expression motion. These 3D points are then mapped onto a 3D face model of arbitrary shape - the model does not have to relate to the subject from which marker tracking was taken. Mapping was done using radial basis functions $(\mathrm{RBF})$. A facial animation system that uses virtual abstract muscle definitions is used to convey expressions in the virtual character. The mapped marker performance is used to drive the animation system. Muscle inverse kinematics update muscle contraction parameters in order to match marker position change to the corresponding vertices on the mesh

The system is designed to be low-cost, using only off-the-shelf hardware. Only a standard PC with Firewire webcams and coloured markers (self-adhesive labels) available from stationery suppliers is needed. This makes the system suitable for end-user environments. 
Marker based motion capture was chosen for its robustness and relatively low computational costs. The animation system supports a physically modelled skin tissue continuum, meaning smooth motion can be represented with fewer markers. The advantages of the presented system lie in the cutting down on creation time by using virtual muscles and dynamic skin model along with its low cost hardware requirements making it suitable for interactive media in enduser environments.

Firstly a brief overview of related work is given in Section 2 The system overview is described in Section [3] along with the development platform and hardware. Sections 4, 6 describe the system in depth. Finally, visual results are given in Section 7 along with a commentary on the advantages and disadvantages of the presented solution.

\section{Related Work}

Virtual characters are now widespread in motion pictures and video-games, and a wide range of animation methods exist [13].

Chai et. al 10 present a markerless vision based system that can generate virtual expressions using a single camera. A face mesh of the test subject is scanned and a database of marker motion capture data is created. Face feature points are then estimated and correlated with data in the motion capture database to create facial expressions on an arbitrary 3D character.

A finite element based physical simulation of facial tissue, created with the visible human dataset, is used to create a parameterised face model in [5]. Marker motion capture data is then used to determine muscle activations of the model. Despite realistic results, the complexity of the model makes it unsuitable for real-time application on end-user hardware.

The Playable Universal Capture system, described in [8, presents a real-time technique for facial animation. The system combines a high-resolution scanned face model which is animated using marker based motion capture data. Texture data is acquired as a video-stream and combined with the model to provide high-quality animation.

Paterson et. al describe a markerless head motion tracking approach using non-linear optimisation [14. The system is able to track head motion through texture alone, however it does not track facial expressions.

The reconstruction of dense 3D data at frame rates capable of capturing facial expression is described by Zhang et. al in [11. Data is acquired through the use of synchronised video cameras and structured light projection.

Motion capture systems are popular in providing animation data to drive these characters. Quality motion data can help give more realistic and immediate results than manual crafting alone. Motion capture is also used in biomechanics and sport performance analysis [17. Still, when fine movement is needed for the face, most motion data has to be refined or applied to crafted keyframe animation in order to look convincing and without errors in a model's surface. The common-place nature of motion capture means that many of the commercial 
systems compatible with many of the leading 3D graphics applications such as Maya and 3ds Max 1,2,

For face motion capture it is common for the user to wear a specially designed helmet with a video camera attached. Optical retro-reflective markers are then tracked and analysed on the computer. There are a wide range of motion capture systems that greatly vary in hardware requirements.

Famous3D sells a single video camera marker based motion capture solution named vTracker [7]. The Vicon MX system uses a remote video sync unit called an Ultranet. This supports up to eight cameras per unit, and these can be stacked to provide 245 cameras per PC. Vicon provides a range of cameras that can be used with the system, as an example, their F40 camera can capture 370 frames-per-second at a full frame resolution of 4 Megapixels 20]. Mova provides a markerless motion capture solution called Mova Contour that uses 44 cameras to carve out 3D space in order to create a face performance. Special phosphorescent makeup that matches the normal flesh-tone in visible light is used to help reconstruction [12].

For character creation, Famous3D provides software to easily animate characters using motion capture data [7]. Face Robot by SoftImage [16] is an advanced software solution for the creation and animation of virtual characters with the ability to incorporate motion capture data to drive the animation, purporting to be faster than tradition solutions by using a soft tissue solver.

A common feature of the aforementioned systems is their high hardware requirement; most of the systems are optically based, with an uncomplicated software element. Although this provides utmost quality in data, these systems are expensive and therefore not applicable for end-user environments; situations where robustness and ease of use are more important than accuracy.

Also, the software environments often have a high learning curve, being targeted at professional animators.

The solution presented in this paper aims at being low-cost and computer vision based, suitable for end-user environments. It has potential applications for light-weight interactive applications and possibly constrained devices where accuracy of results is less important that conveying a visually appealing result.

The usability of stereo cameras for tracking markers has been justified by Woodward et al. 23], providing better robustness and localisation over a mono camera solution, while still maintaining low overall system requirements.

\section{System Overview}

The system comprises three modules: marker tracking, face animation, and the virtual performance creator which links the other two modules. Software was written in $\mathrm{C}++$ and used Microsoft MFC for the GUI and OpenGL for 3D visualisation. The system was developed on a Pentium $43.0 \mathrm{GHz}$ machine with 1 gigabyte of RAM running Windows XP.

Marker Tracking: to make the system practical the marker tracking module captures the stereo view using two off-the-shelf Firewire web-cameras, synchronised 
over the 1394 bus, and provides the calibration of this system. A marker template can be created to track coloured markers through a video stream. 2D marker projections are associated between stereo image frames and these can be triangulated to recover $3 \mathrm{D}$ marker positions. The tracking can the be recorded to file, each frame being time-stamped. The use of a stereo system makes 3D point localisation more robust compared to a single camera system.

Face animation: the facial animation module comprises of a $3 \mathrm{D}$ face mesh that has a predefined virtual muscle animation system. The animation system provides two modes of animation; geometric mesh deformation, and deformation of a physically based skin tissue simulation.

Virtual performance creator: the virtual performance module assigns 3D markers to the model and creates a mapping between the two. Doing so allows any marker configuration and test subject to be used with any mesh that is fitted with the face animation module. Once this mapping is completed, face animation can be driven by the markers through the calculation of muscle inverse kinematics.

\subsection{Camera Specification and Calibration}

A stereo configuration of two Unibrain Fire-i Digital Cameras running over a IEEE-1394a (Firewire) interface was used. Firewire was chosen over USB 2.0 as it has better support for industrial cameras, and easy access and control of IIDC-1394 compliant cameras through the CMU 1394 camera driver and API. The camera operates at 640 by 480 pixel resolution and has a $4.3 \mathrm{~mm}$ focal length - the technical specification of the camera is given in [19].

Tsai's geometric camera calibration [18] was used to estimate the intrinsic and extrinsic parameters of the cameras due to its proved effectiveness in past research. This provides the equations for mapping world coordinates to image coordinates. Once markers have been identified in the stereo image pair, knowledge of the system geometry is necessary for recovering $3 \mathrm{D}$ marker locations through triangulation.

A calibration target was designed consisting of 30 circular markings distributed evenly over its surface. Experiments in reconstructing calibration markings with known true locations have shown that the errors are stable between $40 \mathrm{~cm}$ and $100 \mathrm{~cm}$ from the world origin, with a mean error of approximately 1 $\mathrm{mm}$ and std. of $0.48 \mathrm{~mm}$. However, in practice the delineation and localisation of coloured markers is more prone to noise than the calibration target - noticeable in the jitter seen in recovered marker positions.

\section{$4 \quad$ Marker Tracking}

Marker tracking begins by localising candidate markers within each stereo image. For this experiment, 12 coloured self-adhesive blue markers were used. Their 
locations are determined by using a colour predicate [3]. Once this is done, a marker template can be constructed and then tracked through the video stream. The tracking procedure is given in Algorithm 1 .

The marker template defines the position and number of markers to be tracked within the image and provides robustness against any extra erroneous localisations from being considered. Once a template has been defined, only the areas around the current marker locations need to be processed and searched in, thus reducing computation time.

Markers are placed in facial locations that best represent face motion. The current marker test setup is displayed in Fig. 1] Note that this has a minimalist number of markers acting for experimental purposes and to reduce setup time. A subset of the template markers are designated as anchor points (shown as white points in Fig. 1). These anchors are located where a good estimate of the head rigid motion can be taken. They should not be affected by facial expressions as they are used to define a local head coordinate frame (described in Section 4).
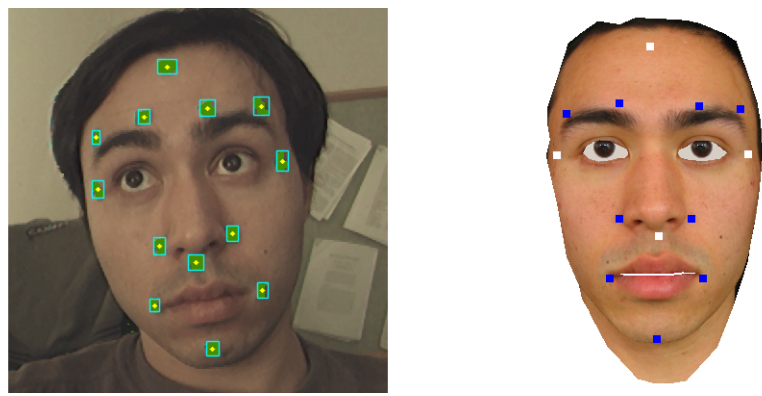

Fig. 1. Marker placement on the face, for the real and virtual cases. White points on the virtual model indicate anchor markers: the forehead, to the outer side of the left and right eyes, and below the nose.

A marker is considered to have disappeared if a new position that is below a certain distance threshold cannot be found - in this case the previous marker position is carried through into the current frame. In practice this works adequately for temporary loss of markers, in the case of an object such as the hand briefly occluding the face when the face is not undergoing substantial movement. Estimation using marker's velocity was found unreliable due to the changing acceleration in marker trajectories as they follow muscular contractions.

Marker correspondence between stereo frames can be found with respect to the scan order and epipolar geometry condition of the stereo cameras. The marker template records the current position of the markers, their number, and velocity. Marker correspondences can then be triangulated to recover the 3D locations.

The presence of noise within the system causes jitter in the triangulated 3D marker positions. Noise sources include optical distortions, imaging noise and 
inaccuracies in the calibration. To stabilise 3D marker positions we apply temporal mean filtering over three frames. This gives a noticeable improvement in the stability of markers, removing a level of jitter.

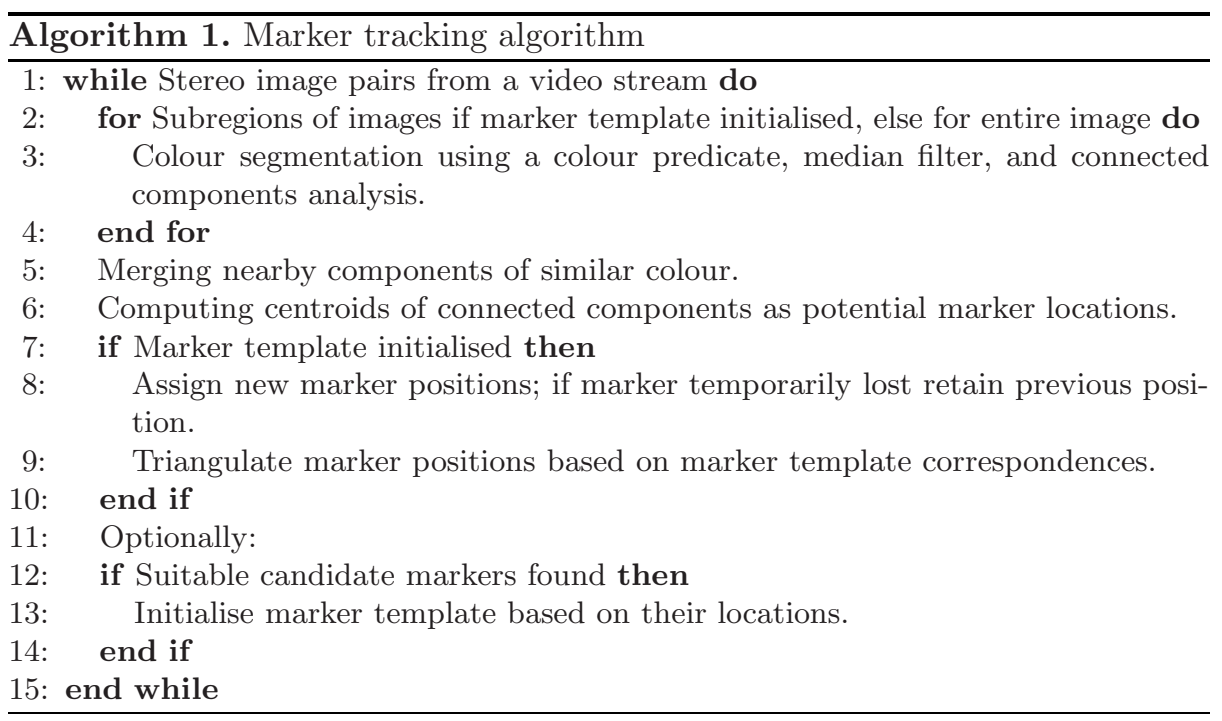

\section{Head Local Coordinate Frame and Reference Marker Positions}

Rigid head motion must be accounted for in order to estimate the non-rigid motion underlying facial expressions. This can be done by constructing a local coordinate system based on the four aforementioned anchor markers described, and shown in Fig. 1. The centre point of the four markers acts as the centre of gravity, or translational component. The four marker points $\overrightarrow{a_{1}}, \overrightarrow{a_{2}}, \overrightarrow{a_{3}}, \overrightarrow{a_{4}}$ are arranged so that the vector from $\overrightarrow{a_{1}}$ to $\overrightarrow{a_{2}}$ is orthogonal to the vector formed from $\overrightarrow{a_{3}}$ to $\overrightarrow{a_{4}}$. These can be used to create the local coordinate frame $R=\left(\overrightarrow{r_{1}} \overrightarrow{r_{2}} \overrightarrow{r_{3}}\right)$ as follows:

$$
\overrightarrow{r_{1}}=\frac{\left(\overrightarrow{a_{2}}-\overrightarrow{a_{4}}\right)}{\left|\overrightarrow{a_{2}}-\overrightarrow{a_{4}}\right|}, \overrightarrow{r_{3}}=\overrightarrow{r_{1}} \times \frac{\left(\overrightarrow{a_{3}}-\overrightarrow{a_{1}}\right)}{\left|\overrightarrow{a_{3}}-\overrightarrow{a_{1}}\right|}, \overrightarrow{r_{2}}=\overrightarrow{r_{1}} \times \overrightarrow{r_{3}}
$$

where $x$ denotes the outer product. The calculation ensures that the coordinate frame is orthogonal.

A neutral expression is recorded to provide default reference marker positions (rest state) within the local head frame. This reference frame can be taken as the first few seconds of marker motion capture where the test subject is asked to keep their face in a neutral expression without facial movement (head movement permissible). These reference marker positions allow for the calculation of divergences from the rest state, and are also used in the RBF mapping procedure (Section 6.1). These divergences are used to create the virtual performance (Section 6). 


\section{Face Animation System}

A face animation system that uses a virtual muscle approach was implemented. 17 virtual muscles are placed in anatomically based positions within a 3D human face model. The choice of face model is conveniently arbitrary since the muscles are defined separately from the face model. They provide an abstract description of a subjects facial expression performance and their usage also means that a face model does not need to be designed with knowledge of a certain marker configuration making it easy to change marker positions.

The face mesh can be deformed in two modes, firstly a simple geometric deformation where vertices are moved independently by the virtual muscles. Secondly, for more realistic facial animation, a physically based deformation approach with a layered tissue model was created. Since the facial tissue is not just a single layer of skin, a mass-spring system was used for the layered tissue model, representing the epidermal, fascial and skull layers. This model is more faithful to the form of human facial tissue - nodes and springs provide a natural behaviour for the skin tissue; stretching and pulling as forces are applied. This means that less markers are needed in order to produce smooth animation over the face surface.

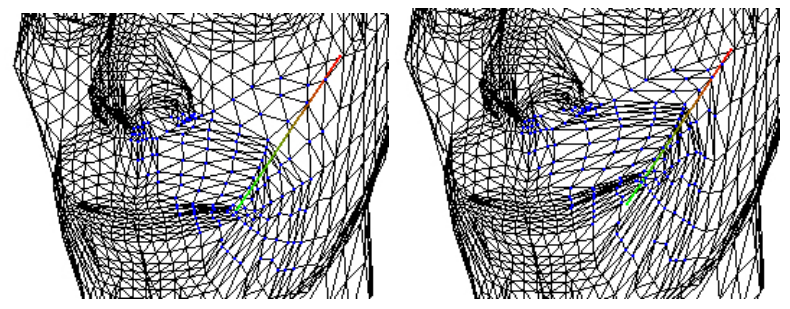

Fig. 2. The face mesh before, left, and after, right, a linear muscle contraction

Two types of muscle were used that apply direct deformation to the mesh in the geometric mode, or apply forces to the skin tissue model in the physically based approach:

- Linear muscle: has an origin where the muscle is attached to the bone, and another point which describes the insertion into the skin tissue. It possesses an angular and radial range. Points that lie within a certain angular zone to the muscle vector and within a certain radial distance from its origin are affected by this muscle.

- Ellipsoid muscle: acting like a string bag; points are squeezed toward the muscle origin. It possesses a radial range and has no angular range. Because of its function, this muscle is also known as a sphincter muscle and can be defined by an ellipsoid, having a major and two minor axes.

In addition, jaw movement was modelled by rotating vertices around a jaw axis. For the physically based system the jaw skull layer was rotated allowing 
the skin tissue to follow along. The complete animation system and formulae is described in depth in 22 .

\section{Virtual Performance Creation}

Once 3D marker positions have been obtained they can be mapped and applied to the face model and animation system described in Section 5 , The local changes in 3D marker positions drive the face muscles through inverse kinematics. This section describes the process of creating a complete virtual performance given a set of $3 \mathrm{D}$ markers and a face animation system.

\subsection{Marker to Model Correspondence}

A design goal was to allow for the use of any arbitrary 3D human face model to be used for a particular set of motion capture data. This allows for an automated solution and the ability to retrofit existing models, along with the system being able to drive a wide variety of face models that do not have to be human in nature. In order to do this, mapping of markers into a new face space was performed by using RBF where the required correspondences between markers and vertices of the mesh are entered manually. This procedure needs only be done once for a certain marker configuration and face model, as the marker template, marker correspondences, and RBF mapping coefficients can be archived for further use.

RBF Mapping: For the case of a non-rigid mapping between datasets, where one dataset is of a face model and the other of 3D marker positions, two criteria must be met. Firstly a set of correspondences is needed between datasets so that there is knowledge of how the non-rigid mapping should proceed. Secondly, the vertex distribution and resolution of the face model must be preserved. This makes a data interpolation strategy appropriate. Radial basis functions are a form of interpolant with desirable smooth properties 9, 15.

Considering the $1 \mathrm{D}$ case, the goal of interpolation is to analytically approximate a real valued function $g(x)$ by $s(x)$ given the set of values $g=\left(g_{1}, \ldots, g_{N}\right)$ at the distinct points $X=\left(x_{1}, \ldots, x_{N}\right)$. This is naturally extended for this research to the orthogonal $3 \mathrm{D}$ spatial dimension, where a function that maps spatial points is required. In practice, the two sets of data are the points that are chosen to correspond between the 3D reference markers $X$, as calculated in Section 4 and the virtual face mesh vertices $g$.

In general a 3D RBF has the form: $s(x)=p(x)+\sum_{i=1}^{N} \lambda_{i} \omega\left(\left|x-x_{i}\right|\right), \quad x \in$ $R^{3}$, where $p$ is a polynomial of degree at most $k, \lambda_{i}$ is a real valued weight, $\left|x-x_{i}\right|$ specifies the Euclidean distance between the points $x$ and $x_{i}$ and $\omega$ is a basic function [9]. The chosen sets $X$ and $g$ will generally be much less than the number of points contained in the source dataset.

This mapping is done once based on the neutral reference marker frame and the neutral face mesh. The marker performance can then be translated into 
the virtual mesh local coordinate frame by giving the $3 \mathrm{D}$ marker positions to the RBF interpolant; the resulting output positions can then be measured as divergences of marker vertices from the mesh rest state.

To find the RBF interpolant for a given set of correspondences $f$ and $X$, it is necessary to estimate the polynomial term $p$ and the real valued weights $\lambda_{i}$. This under-determined system of linear equations was solved using standard least-squares estimation.

The benefit of using a scattered data interpolation approach is the abstraction from the specifics of the input data. Only point locations need to be dealt with.
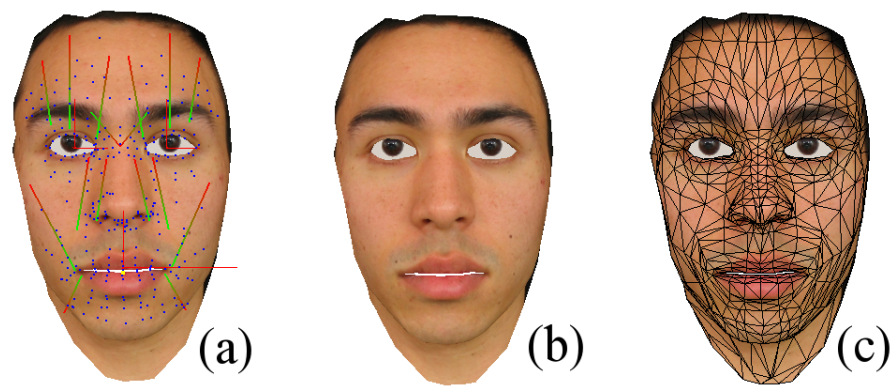

Fig. 3. The face animation system showing muscle placements (a), texture only (b), and the underlying mesh (c)

\subsection{Driving Face Animation Through Marker Motion}

At this stage the system is able to measure marker divergences from a rest frame mapped into a virtual face model's local space. Each marker is associated with a vertex of the mesh. Upon initialisation the muscles that influence each marker must be found. This is easily done as each muscle has an area of influence. When a marker moves we want to update the virtual muscles to deform the mesh in order to make the correspondent vertex on the mesh be as close as possible to the marker. In order to do this, inverse kinematics was used. It is widely used in robotics and as a tool for animating articulated figures in order to reduce the amount of data needed to be specified each animation frame [21.

Muscle Inverse Kinematics Using Marker Positions: Inverse kinematics is the calculation of parameters for a kinematic chain in order to meet a desired goal position $\vec{g}$, when starting from an initial position $\vec{e}$. Applied to this research, the desired end position is the $3 \mathrm{D}$ marker position as tracked by the vision system described in Section 4, and the initial (current) position is considered to be the vertex on the mesh corresponding to the marker. The kinematic chain consists of a set of joints: the muscles or jaw affecting a certain marker. Since we only deal with contraction values each muscle, and the jaw, possesses 1-DOF (Degree 
of Freedom) 1 . Only the start and end locations of the marker need to be planned for the animation. This is a much simpler way to generate a performance based on 3D motion capture when opposed to forward kinematics, where each muscle contraction value must be determined.

Considering a single vertex and marker correspondence, the inverse kinematics problem is to compute the vector of muscle DOFs, $\vec{\Phi}=\left\{\phi_{1}, \phi_{2}, . ., \phi_{N}\right\}$, from a target marker position: $\vec{\Phi}=f^{-1}(\vec{e})$, where $f$ is the forward kinematics map. Hence, for inverse kinematics an update value, $\Delta \vec{\Phi} \approx J^{-1} \cdot \Delta \vec{e}$, is sought after.

A fast and simple iterative gradient descent solution was chosen that uses the Jacobian transpose of marker position with respect to muscle contraction (or jaw rotation around its axis) 4. This method is computationally inexpensive since there is no inversion of the Jacobian matrix. It also localises computations since the degrees of freedom can be updated before the entire Jacobian is computed:

$$
\Delta \vec{\Phi}=J^{T} \Delta \vec{e}
$$

Intuitively, smaller step sizes are needed with respect to the complexity of marker movement. The side effect of this is an increase in the number of iterations required to find a solution.

Calculating the entries of the Jacobian matrix is straightforward. Entries of $J$ specify DOFs and depend on the type of joint - in this case muscular contraction is a linear joint and jaw rotation an angular joint.

For a muscle DOF a vertex $\vec{e}$ is draw toward the muscle's origin $\vec{o}$. The possible values for the DOF is bounded as muscles have a physical contraction range. A change in the DOF $\phi$ gives a movement along this direction:

$$
\frac{\partial \overrightarrow{e_{i}}}{\partial \phi_{j}}=(\vec{o}-\vec{e})
$$

Consider the $j$ th joint of a kinematic chain to be a 1-DOF rotational joint which models jaw motion. Its entry in the Jacobian measures how the end effector, $e$, changes during a rotation about its axis:

$$
\frac{\partial \overrightarrow{e_{i}}}{\partial \phi_{j}}=\overrightarrow{a_{j}^{\prime}} \times\left(\overrightarrow{e_{i}}-\overrightarrow{r_{j}^{\prime}}\right)
$$

The rate of change of the vertex $e$ position (the velocity) is in a tangential direction to the rotation axis $\overrightarrow{a_{j}^{\prime}}$, scaled by the distance of the axis $\overrightarrow{r_{j}^{\prime}}$ to $\vec{e}$.

The step $\Delta \vec{e}$ is given as:

$$
\Delta \vec{e}=\beta(\vec{g}-\vec{e})
$$

\footnotetext{
${ }^{1}$ Degrees of Freedom (DOF) refer to the set of animatable parameters within the virtual face, where the animatable parameters are the muscle contraction values and jaw rotation. In generality, joint DOFs are a common example, i.e. three orientation parameters and a position gives 6-DOF. Collectively all of these parameters describe the system state of the virtual muscles. Their change provides the virtual character performance.
} 
The scale factor $\beta$ is used to limit the step size since we are dealing with non-smooth functions. Since smaller step sizes must be taken when the number of muscles increases the value of $\beta$ could be tuned for each marker.

Pseudo-Code for Muscle Inverse Kinematics. Pseudo-code is given in Algorithm 2 markerCount gives the number of markers being tracked and affectedMuscleCount $(j)$ specifies the number of muscles affecting a particular marker $j$.

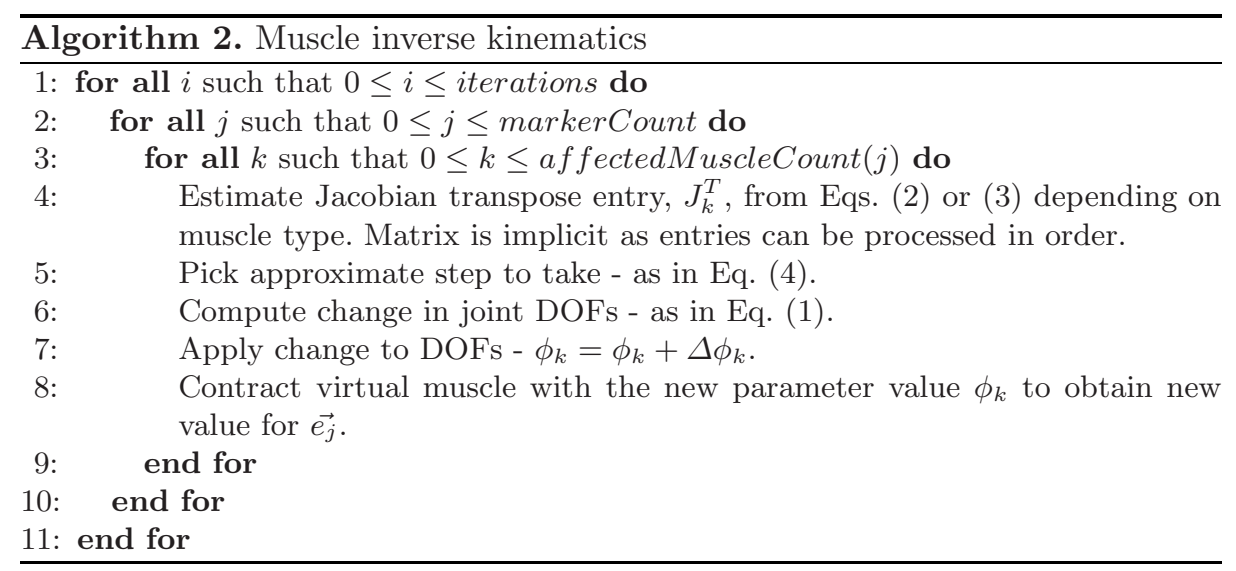

\subsection{Expression Estimation}

It has been found that sadness, anger, joy, fear, disgust and surprise are six universal categories of facial expression that all cultures can recognise [6]. Falling within these categories are a range of possible intensities and variations in expression detail. Face expressions can be hand crafted using the face animation system and adjusting muscle contraction parameters.

\section{Experimental Results and Conclusions}

Our experiments show that the proposed system is capable of reproducing face expressions from marker motion, a selection of results are shown in Fig. 4.

The attraction of using a marker based system is its robustness, and relatively low computational costs and formulation in relation to markerless motion capture approaches. This system provides an automatic face performance from marker data and can therefore operate on a wider variety of face models and applications - avoiding the need for time consuming model creation. Also, virtual muscles are easier to set up than keyframing and are anatomically based. Setup costs are low compared to other motion capture solutions, however placing markers on a face is time consuming, especially if placement needs to be precise and repeatable. This points to a future direction of research involving markerless 

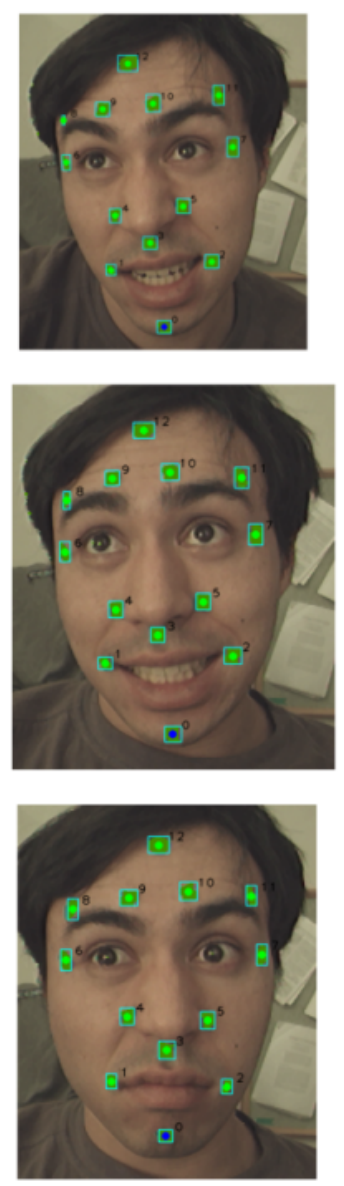
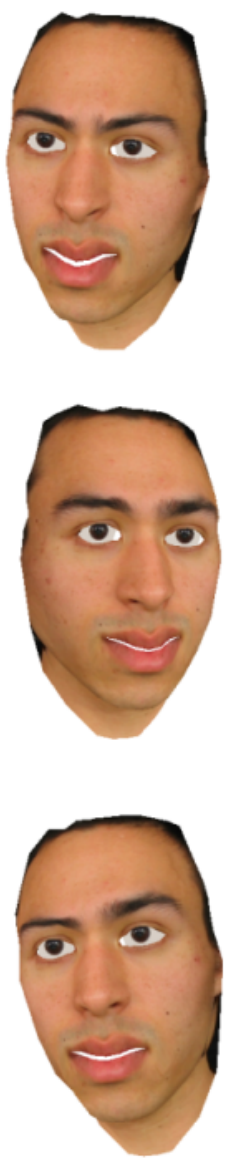

Fig. 4. Tracked markers on a subject's face (left column). Synthetic performance results (middle column). An angry expression (right), marker divergences from the neutral frame is indicated by lines.

motion capture. In doing so, the trade-offs between an automatic process and accuracy need to be investigated.

Noise in the detection of 2D marker positions affect the quality of 3D triangulation. Illumination conditions affected the detection of the coloured markers within the image and it was important to have uniform and controlled illumination. The robustness of marker detection would need to be improved if constraints on an end-users environment were to be relaxed. To this end, improvement in the localisation of markers and their triangulation will be investigated. Also, it was found that noise and not rigid enough positions of the anchor points created some jitter in the local coordinate system. A better means of deriving this frame should be investigated. A possible solution is for the user to wear a head band 
with markers on it. This would be more stable than applying representative anchor markers on the face.

RBF provides an effective means of mapping markers to a face model. There is no restriction on the face model used and it does not have to represent the test subject. RBF is inexpensive and the mapping coefficients for a particular system setup need only be estimated once based on a neutral face expression.

The quality of animation greatly depends on the quality of the animation system and how well it can mimic marker motion. The physically based skin system provides better animation when a low number of markers is used as it treats the mesh as a skin continuum, making it of benefit for creating novel animations. However, this does not guarantee that the final animation is representative of the actual expressions performed by a human test subject.

Placement of additional markers will be looked at to improve animation this will possibly mean more face muscles will be required around very dynamic areas of the face, such as the mouth region. Since the markers can be transferable between models and animation systems, it could be used to drive alternate animation systems, e.g. key-frame based. A hybrid of the two could be combined.

A better inverse kinematics scheme will be pursued to handle fast marker movement when a marker is affected by a large number of muscles and an improved face model, with a complete head and ears, will also be tested.

The system will be tested on higher quality video cameras, providing better triangulation of 3D markers and higher acquisition rates. Upon writing, specially designed reflective motion capture markers have been recently acquired. They should allow better detection of markers giving less noise in the tracked 3D marker positions.

A database of expressions could be created in order to estimate the validity of synthesised expressions and their recognition. An investigation on the impact of the number of markers on the quality of animation will be undertaken, this will determine the expressive ability of the face animation system and the determination of a balance with computational time.

\section{References}

1. Autodesk. 3ds max (2007), http://www.autodesk.com/3dsmax

2. Autodesk. Autodesk maya (2007), http://www.autodesk.com/maya

3. Barton, G., Delmas, P.: A semi-automated colour predicate for robust skin detection. In: Proc. Image and Vision Computing New Zealand, pp. 121-125 (2002)

4. Baxter, B.: Fast numerical methods for inverse kinematics (2007), http://billbaxter.com/courses/290/html/index.htm

5. Neverov, I., Sifakis, E., Fedkiw, R.: Automatic determination of facial muscle activations from sparse motion capture marker data. In: ACM Transactions on Graphics (SIGGRAPH Proceedings), pp. 417-425. ACM Press, New York (2005)

6. Ekman, P., Friesen, W.V.: Constants across cultures in the face and emotion. Journal of Social and Personality Psychology, 124-129 (1971)

7. famous3D. famous3D (2007), http://famous3d.com/

8. Montgomery, J., Borshukov, G., Hable, J.: GPU Gems 3 - Playable Universal Capture, ch. 15, pp. 485-504. Addison-Wesley Professional, Reading (2007) 
9. Cherrie, J., Mitchell, T., Fright, W., McCallum, B., Carr, J., Beatson, R., Evans, T.: Reconstruction and representation of $3 \mathrm{D}$ objects with radial basis functions. In: ACM SIGGRAPH, pp. 67-76. ACM Press, New York (2001)

10. Xiao, J., Chai, J., Hodgins, J.: Vision-based control of $3 \mathrm{~d}$ facial animation. In: Proc. of the ACM SIGGRAPH/Eurographics Symposium on Computer Animation, ACM Press, New York (2003)

11. Curless, B., Zhang, L., Snavely, N., Seitz, S.: Spacetime faces: High-resolution capture for modeling and animation. In: ACM Annual Conference on Computer Graphics, pp. 548-558. ACM Press, New York (2004)

12. Mova. Mova (2007), http://www.mova.com/

13. Noh, J., Neumann, U.: A survey of facial modeling and animation techniques. In: USC Technical Report, University of Southern California (1993)

14. Paterson, J., Fitzgibbon, A.: 3D head tracking using non-linear optimization. In: Proc. of the British Machine Vision Conference 2003, vol. 2, pp. 609-618 (2003)

15. HomePage of Applied Research Associates NZ Ltd (ARANZ): Interpolating scattered data with RBFs (2007), http://www.aranz.com/research/modelling/theory/rbffaq.html

16. Softimage. Face robot (2007), http://www. softimage.com/products/facerobot/

17. Motek Motion Technology. Motek - motion technology (2007), http://www.e-motek.com/

18. Tsai, R.Y.: A versatile camera calibration technique for high-accuracy 3D machine vision metrology using off-the-shelf TV cameras and lenses. IEEE Journal of Robotics and Automation, 323-344 (1987)

19. Unibrain. Unibrain fire-i digital camera (2007), http://www.unibrain.com/Products/VisionImg/Fire_i_DC.htm

20. Vicon. Vicon (2007), http://www.vicon.com/

21. Welman, C.: Inverse kinematics and geometric constraints for articulated figure manipulation. Master thesis, Simon Fraser University (1993)

22. Woodward, A., Delmas, P.: Towards a low cost realistic human face modelling and animation framework. Proc. Image and Vision Computing New Zealand, 11-16 (2004)

23. Woodward, A., Delmas, P.: Computer vision for low cost 3-D golf ball and club tracking. Proc. Image and Vision Computing New Zealand (2005) 